\title{
COMPETENCIAS COGNOSCITIVAS \\ Y EVALUACIÓN
}

\author{
Roymán Pérez Miranda, Rómulo Gallego Badillo \\ Universidad Pedagógica Nacional.
}

\begin{abstract}
Education towered on competences is a major issue now days. It is, adecuate therefore, to give proper consideration to what is understood by comeptences, and the epistemological, educational and didactic frame from which said compenhension stems. This comprehension senders possible an evaluation that will include the type of results being sought, what to do which them, as well as the intentionality of the evaluation. This paper intends to face some considerations and anwers an this respect.
\end{abstract}

\section{RESUMEN}

Se habla hoy de una educación centrada en competencias, razón por al cual es indispensable discutir qué es lo que se entiende por ellas y desde qué marcos epistemológicos, pedagógicos y didácticos se produce esa comprensión; desde dicha comprensión es que se hace factible plantear una evaluación explicitando de antemano ¿Qué tipo de resultados se persigue? ¿Qué hacer con ellos? y ¿Cuáles las intencionalidades de esa evaluación? En el presente texto se abordan algunas consideraciones y respuestas al respecto.

\section{INTRODUCCIÓN}

Durante los años setenta, en Colombia, se puso en práctica un plan de estudios vertebrado por una concepción epistemológica, pedagógica y didáctica de objetivos, cuyos fundamentos hay que encontrarlos en el empiropositivismo y en la psicología behaviorista, en particular la del conductismo operante. Esta precisión viene para subrayar que la palabra objetivo adquirió así, una connotación particular, distinta de la que podría encontrarse en cualquier diccionario general.

La posición conductista comenzó a perder dominancia a partir de la discusión que $\mathrm{N}$. Chomsky (1965) hace del texto de Skinner, sobre la conducta verbal, en donde intentó explicar el aprendizaje de la lengua según su fórmula E-OR.; algo a lo que racionalmente se opuso Chomsky, argumentando que tal fórmula no daba cuenta de la naturaleza creativa del hablar humano. En general, propuso, en su defecto, que era necesario construir una nueva teoría cognoscitiva sobre la adquisición de la lengua y para lo cual introdujo el concepto de competencias, que definió como capacidades y disposiciones para la interpretación y la actuación. 
Hay que destacar el empleo, en esta definición, de las categorías capacidad, disposición, interpretación y actuación. En relación con las dos primeras y tomando distancia conceptual y metodológica del autor, se piensa que alude tanto a inteligencia como a actitudes respectivamente (Gallego Badillo R., 1999), por lo que se hace necesario discurrir en torno a aquello que se entiende por la una tanto como por la otra. En cuanto a interpretación y actuación se constituyen en un par de categorías que igualmente merecen ser analizadas.

Sobre la actuación habría que acudir al lenguaje del teatro para precisar, en primer lugar, que el ser humano actúa siempre dentro de un escenario particular, si se quiere, en términos reduccionistas, en el interior de situaciones específicas. En segundo lugar, que toda actuación deviene de una interpretación, ya sea de esa situación aludida, de un texto o de una estructura conceptual y metodológica que delimita por qué y cómo actuar en el marco del libreto de que se trate. Puntualícese que, dentro de esta connotación toda actuación, así considerada, es un representar desde sí para los otros, con los otros y en el seno de los otros. Precisando lo anotado, hay que sostener que cada campo o cada situación determina de una u otra manera el cómo de ese representarse y en consecuencia de esa representación. En el caso, por ejemplo, de las ciencias experimentales, las respectivas comunidades de especialistas tanto como los saberes por ellas construidas son las que exigen tal especificidad (Toulmin, S., 1976).

Dejando de lado a N. Chomsky, anótese, además, que en contra del conductismo emergió el programa de investigación "Epistemología Genética" formulado por J. Piaget, investigador éste que desde una mirada constructivista, se ocupo del desarrollo de la inteligencia en el niño; programa en el que postuló, en contra del behaviorismo, la existencia de unas estructuras mentales con sus lógicas operatorias correspondientes, necesarias para auscultar metódicamente y postular, como una aproximación, en qué estadio de ese desarrollo se encontraban infantes particulares.

La perspectiva investigativa que abre Piaget y con el apoyo de las elaboraciones epistemológicas constructivistas, en el campo de las ciencias experimentales se emprendieron distintos programas de investigación tendientes a estudiar el proceso de construcción de actitudes positivas, por parte de los estudiantes, hacia esas ciencias. En este proceso y, dada la reconceptualización que hace J. Piaget, de la inteligencia humana, se empezó a dejar de lado tanto ideas genetistas como la noción de aptitud.

Las elaboraciones teóricas y los resultados obtenidos de tales investigaciones, condujeron en principio, a descartar el conductismo y el problema de sus objetivos instruccionales, para formular proyectos educativos centrados en logros. Subráyese que detrás de la concepción de logros se hallan otras aproximaciones epistemológicas, pedagógicas y didácticas, a la vez que la intencionalidad es justamente la elaboración de actitudes positivas, tomando para tal efecto los contenidos curriculares como base y pretexto. Así, la idea de enseñanza y de aprendizaje como transmisión y repetición al pie de la letra (Ausubel, D., Novak, J. D. y Hanesian, H. 1983), fue sustituida perla de aprendizaje significativo primero y, luego, por las sucesivas reconceptualizaciones del aprendizaje como cambio, siendo la última de estas el del cambio conceptual, metodológico, estético, actitudinal y axiológico (Gallego Badillo, R, y Pérez Miranda, R. 1998), hoy, en la perspectiva de un aprendizaje total (Gallego Badillo, R. Pérez Miranda, R. y Torres de Gallego, L. N. 1997).

Las anotaciones anteriores dejan sentado, de manera clara que el concepto de logros tiene unos fundamentos precisos de naturaleza epistemológica, pedagógica y didáctica, 
por lo que, al igual que el de objetivos, no puede ser especificado desde una consulta cualquiera a un diccionario general. De otra manera, y aun cuando semánticamente objetivos y logros pueden interpretarse como equivalentes, en este contexto precisado, no son lo mismo.

Se considera que en el ámbito colombiano, cuando se pensó introducir el cambio de una educación por objetivos a otra vertebrada por logros, no hubo la suficiente y rigurosa discusión, por lo que muchos profesores y profesoras terminaron reduciendo esos logros a objetivos, preocupados por la transmisión y repetición de contenidos y centrando el análisis en el problema de en qué tiempo verbal se formulaban tales logros.

En la actualidad se está en el inicio de la necesidad de formular proyectos curriculares para la construcción de competencias cognoscitivas (Gallego Badillo, R. y Pérez Miranda, R. 1998); comprensión de las cuales exige, de igual manera, una explicitación de las concepciones epistemológicas desde las cuales se proponen, lo que reclama, necesariamente la puesta sobre la mesa de las teorías y modelos pedagógicos y didácticos con los cuales esa concepción es coherente. Agréguese, como ha quedado dicho, a partir de una mirada sobre la definición que sobre ellas hace N. Chomsky, que se requiere también discutir cuáles son las ideas de inteligencia y de actitud que manejan aquellos que hacen dicha explicitación y ponen de presente esas teorías y modelos pedagógicos y didácticos desde los cuales van a llevar al aula la formulación por competencias y la praxis de los proyectos curriculares correspondientes.

Acotado lo anterior, es indispensable destacar que esta educación direccionada por la construcción de competencias exige un cambio en profesores y profesoras. Es así, por cuanto la propuesta de cambio de dirección y de intencionalidad de la formación de las nuevas generaciones implica una transformación de las concepciones sobre el currículo, modificando lo ya planteado históricamente, por otra versión que sea congruente con esa propuesta de construcción de competencias: demanda un cambio en el proyecto curricular que ha venido predominando y en las actitudes, no sólo de los docentes, sino también de los estudian-tos y de las directivas académico administrativas de las instituciones escolares.

\section{COMPETENCIAS COGNOSCITIVAS}

La adjetivación de cognoscitivas establece ya una diferenciación en relación con aquellas ideas desde las cuales se mira a las competencias como simples habilidades y destrezas, en el contexto de un transmisionismo repeticionista en el que ellas, como tales, se ponen a punto por procesos de entrenamiento. No es que aquí se piense que tales habilidades y destrezas no sean necesarias, lo que ocurre es que ellas han de inscribirse como resultado de un proceso de cognición que las hace, en consecuencia, indispensables. En otras palabras, primero está lo cognoscitivo, como objeto de discusión epistemológica, pedagógica y didáctica, para luego entrar a pensar de qué manera esa cognición determina, en los campos particulares en los que ella se despliega, qué clases de habilidades y destrezas requiere para continuar reconstruyéndose ella misma.

Volviendo al calificativo, es imperante destacar que lo cognoscitivo es una actividad; esa que caracteriza al ser humano como un sujeto cognoscente que vive interrogándose acerca de su propio conocer como de la admisibilidad, tanto para él como para el colectivo al cual pertenece, de aquello que es producto de tal actividad; resultados que, desde la perspectiva kantiana, ha de ser interpretado como saber, en el sentido de que ese saber 
es un arreglo de la razón consigo misma, razón que se halla en la base de ese conocer que, como ha quedado estipulado, puede ser expresado, en términos de preguntarse, responderse y examinar tanto las preguntas como las respuestas elaboradas.

Si lo cognoscitivo es una actividad, entonces, cabe atribuirle a ella una dinámica; atribución que más allá de una simple expresión cotidiana, exige necesariamente posiciones rigurosas, en cuanto a aquello que se entiende por dinámica. De ser así, hay que acudir a la fisicomatemática, puesto que son los miembros de esta comunidad de especialistas quienes se han encargado de estudiar, dicho problema. En este sentido han propuesto, hasta el presente, la existencia teórica de dos dinámicas: de los sistemas no lineales y la de los lineales (Hayles, N. k. 1990).

Si la actividad cognoscitiva sigue una dinámica lineal, entonces se comporta como el reloj, que es el ejemplo más significativo de tal sistema. En esta perspectiva de atribución habría que decir, con $\mathrm{H}$., Poincaré, que sigue esquemas repetitivos y obedece pautas discernibles, por lo cual no podría ser ella creativa, en virtud de que no rompería la simetría temporal y, por tanto, lo que ella es en su actualidad, sería una copia de lo que fue ayer, y lo que sería mañana un calco de su presente (Prigogine, I, y Stengers, I., 1990), Si así fuera, no se encontrarían fundamentos para explicar la historia de las ciencias experimentales (Kuhn, T. S. 1972), la creación de nuevas versiones sobre el mundo y todos los cambios conceptuales y metodológicos que se han sucedido a lo largo de dicha historia. En otras palabras, el ser humano actual no sería otro, en términos de saber, que el mismo del paleolítico. Para conocer la naturaleza creativa de esa actividad cognoscitiva se requiere una dinámica no lineal

Desde la idea de que las competencias son de naturaleza cognoscitiva, se subraya aquí que hay que pensarlas en el horizonte de sentido de que se enclavan en la creatividad que, como ya se dijo es indispensablemente no lineal. Habría que sostener, además, que tales competencias son construcciones de cada ser humano en comunidad, en el interior de escenarios preparados para tal efecto; algo con la cual se precisa que nadie nace programado para desarrollarlas, perfeccionarlas o desplegarlas. Ligando esta observación a las actuales concepciones pedagógicas y didácticas en torno a la enseñanza y al aprendizaje de las ciencias experimentales, por ejemplo, se propone, en consecuencia, que tales competencias, como intencionalidad curricular, se inscribe en un proceso de sucesivas reconstrucciones y construcciones. Es así, por cuanto se admite que cada estudiante, desde su inmersión en el saber común y cotidiano, tanto como a partir de sus experiencias de formación en el sistema educativo institucional, ha de haber elaborado unas competencias, cualesquiera ellas sean, que deben ser objeto de trabajo pedagógico y didáctico para sus correspondientes reconstrucciones.

\section{LAS DIMENSIONES DE LAS COMPETENCIAS}

Apoyándose en Piaget, en cuanto a la construcción individual de la inteligencia, en la relación dialéctica individuo-comunidad (Piaget, J. 1983), tanto como en el problema de las actitudes (Gallego Badillo, R. y Pérez Miranda, R. 1999), se sostiene aquí que la construcción de las competencias, y aun cuando suene pleonástico, por la calificación que se les ha dado, es cognoscitiva, ya que nadie puede construir competencias al respecte sino en el interior de un saber determinado, en la medida en que los procesos educativos le dan la oportunidad de sumergirse en discusión sobre la estructura conceptual y metodológica del mismo, en las razones por las cuales ese saber ha sido admitido históricamente, y por qué la comunidad respectiva lo acogió como una propuesta de 
mayor y mejor productividad. Esta dimensión especifica y pone en tela de juicio cualquier alusión a una enseñanza y a un aprendizaje centrado en la transmisión y la repetición de contenidos curriculares.

Ese sumergirse en un saber determinado conlleva necesariamente la construcción de actitudes positivas hacia el mismo, la cual trae consigo la elaboración de una afectividad, también positiva, como la segunda dimensión que alimente ese dedicarse por encima de las dificultades, en principio, que la comprensión del mismo exige. Dígase que esa afectividad positiva es un enamorarse de aquello que ese saber explicita, de la manera como lo hace y de las exigencias que le demanda para llegar a una satisfacción que el dominio del mismo le genera. Piénsese, desde Freud que lo hace su objeto de deseo y posesión.

La tercera dimensión de las competencias reclama la conceptualización de que ellas son actuacionales, o sea, se expresan en realizaciones o desempeños concretos en escenarios específicos; precisión esta que, en principio, podría servir de base para cualquier proyecto de evaluación; todo porque es aquí, en donde emerge aquello que la idea de habilidades y destrezas recoge; las cuales, al respecto, requieren de contextualizaciones que dependen de ese saber en el cual se reconstruyen y construyen las competencias y en el que, por afectividad, se aspira a ser competente; aspiración que para ser explicitadas necesita de la categoría de pertenencia; en otras palabras, quien decide ser competente en un saber, es aquel que desea pertenecer a la comunidad de especialistas que lo sustenta.

Pero he aquí que, en cuanto a lo actuacional, no puede ser interpretado como una dimensión aparte y separada de lo afectivo y de lo cognoscitivo, ya que estas últimas son a su vez eminentemente actuacionales y se realizan concomitantemente en las particularidades evaluativas que la determinan. En otras palabras, esas tres dimensiones adquieren su fundamentación en la relación holística que hace que cada una de ellas no pueda ser comprendida sin las otras; podría pensarse en que la actuacionalidad inherente a cada una de tales dimensiones es la que las liga, aun cuando la actuacional propiamente dicha se mire como un realizar concreto y objetivable que requiere de unas habilidades y destrezas mecánicas. En todo caso, y siguiendo el orden del discurso, esa dimensión actuacional se desprende de lo correspondiente de las dimensiones cognoscitiva y afectiva: lo actuacional es cognoscitivo y afectivo al mismo tiempo.

\section{LAS SUBDIMENSIONES}

Para plantearlas, se acude a la propuesta de un aprendizaje como cambio conceptual, metodológico, estético, actitudinal y axiológico, por lo que habría que formular para cada una de las dimensiones anotadas, cómo cada una de ellas se realiza, precisamente en estas subdimensiones que le postula hipotéticamente, tal propuesta de aprendizaje. Aun cuando se suele ser reticentes a dar ejemplos, en virtud de que ellos, en sus concreciones suelen polarizar las reflexiones, la pregunta que habría que entrar a resolver es aquella que indaga por la manera como, por ejemplo, una unidad didáctica, como la de los números naturales, se puede especificar en términos cognoscitivos, afectivos y actuacionales, para pasar, seguidamente, a explicitar cómo en la unidad didáctica referida, se realiza, igualmente, cada dimensión, en lo conceptual, lo metodológico, lo estético actitudinal y lo axiológico. Este ejercicio, eminentemente intelectual, es aquel que le permite a cada profesor y profesora comenzar a cuestionarse qué es aquello que entiende por competencias, porque es él el que, en las subdimensiones que se puntualiza, 
en las que puede examinarse en torno si ha o no comprendido la nueva propuesta educativa.

Precisando lo anotado, dígase que una vez acordada una competencia cognoscitiva para la realización de un proyecto curricular específico, ha de discurrirse cómo ella se hace aparecer teóricamente en sus dimensiones cognoscitiva, afectiva y actuacional. Luego de este ejercicio, si se admite la propuesta aquí seguida, ha de entrarse a elucidar cómo cada una de ellas se hace emerger en la naturaleza que se les atribuye como conceptual, metodológica, estética, actitudinal y axiológica; atribución tal que, de nuevo, las hace interactuante de constituyentes de una totalidad holística. Se precisa aquí que es necesario traer a cuento a los estudiantes, esto es, a plantear los problemas pedagógicos y didácticas, tanto como curriculares, que la centración de reconstrucción y construcción demanda, en términos metodológicos y no simplemente de una serie de algoritmos para la praxis educativa consecuente.

\section{INTERPRETAR, ARGUMENTAR, PROPONER}

En este numeral, por lo determinado de las metas conceptuales y metodológicas, desde el punto de vista epistemológico, pedagógico y didáctico, que el examen del Instituto Colombiano para el Fomento de la Educación Superior, ICFES, obliga, se entra ahora a analizar las razones por las cuales se propone, como objeto de evaluación, estas tres competencias ¿Por qué razones son tres? ¿De qué manera están relacionadas unas con otras? ¿Por qué tres competencias en especial? ¿Podrían ser otras?

Al respecto de cualquier pregunta acerca de las razones por las cuales se examina en tres competencias y por qué sólo tres, pueden ser objeto pedagógico y didáctico de un proyecto curricular, en la acotación temporal que él determina, dígase, que no hay respuesta. A lo mejor habría que acudir para tal efecto, al sentido común que afirma aquello que 'quien mucho abarca poco aprieta'. Lo cierto es que para cualquier proyecto curricular que busque como horizonte de sentido la reconstrucción y construcción de competencias, especificadas de antemano, todas las estrategias pedagógicas y didácticas y las planificaciones al respecto, han de orientarse en tal dirección. Esto es, que ese proyecto no puede dividir en el tiempo, o por periodo o por año de escolaridad que establece, que en un momento de ese desarrollo ha de dedicarse a una competencia en particular para la otra otro tiempo y así sucesivamente. En el mismo sentido, asignar espacios pedagógicos y didácticos que, por ejemplo, imitarlos a cuarenta y cinco minutos, podría decirse que en los primeros quince minutos se ocupará de una competencia, en los siguientes de la siguiente competencia y en lo falta de la tercera.

La epistemología, la pedagogía y la didáctica de las competencias cognoscitivas por un acuerdo que valdría investigar, ha concluido que esas tres precisadas, están ligadas unas con otras, hasta el punto de que teórica y prácticamente se codeterminan, lo que hace del proyecto curricular una unidad holística; esto es, la competencia $A$ no es factible sin la competencia $B$, y esta no lo es sin la $C$, por lo que la $C$ no tiene sentido sin la $A$ en las formas como se concretan en sus dimensiones y se precisa en las subdimensiones que se le han atribuido a cada una de ellas.

Es en esta precisión, en la que hay que entender que la competencia interpretativa, la argumentativa y la prepositiva, dentro de la prueba del ICFES, no pueden separarse a la vez que cada una de ellas precisar los desempeños que se establecen y que obligan a las discriminaciones respectivas. Para tal efecto, por ejemplo, cada estudiante puede hacer 
una interpretación aceptable de aquello que en el saber respectivo cada comunidad de especialistas ha hecho. Pero esa interpretación idiosincrática, en principio, no puede ser asumida como una propuesta novedosa desde lo que él piensa, es mejor que las otras construidas por las miembros de su colectivo aula.

Lo que se espera es que cada interpretación de aquello que es objeto de estudio de cada colectivo aula, sea indispensablemente una interpretación novedosa y que cada proponente argumente en forma rigurosa que esa interpretación que hace, es la más aceptable, aun cuando el lenguaje en el cual esa interpretación se expresa no sea el propio de la comunidad de especialistas, que por tal efecto, se constituye en el referente de evaluación; lo que exige, sin lugar a dudas, que aquel que ejerce de evaluador pertenezca a la comunidad de especialistas en el saber en el que la interpretación se da. Este es el problema.

\section{EL CASO DE LA EVALUACIÓN}

Según lo puesto en discusión, parecería ser claro que ninguna institución educativa podría adentrarse en un proyecte educativo de reconstrucción y construcción de competencias cognoscitivas si no propicia unas discusiones rigurosas en torno a lo que ellas significan y la apertura pedagógica y didáctica que obre como actuación docente, estudiantil y comunitaria.

En efecto, el concepto de competencias cognoscitivas debe considerarse de carácter epistemológico, pedagógico y didáctico el cual no puede resolverse desde esquemas ya dados, que buscan repetir la praxis profesional de educadores y educadoras en el interior de, puesto que se está frente a una propuesta educativa que persigue otros horizontes. De hecho, en lo que se puntualiza aquí es en la convicción de que un currículo centrado en la reconstrucción y construcción de competencias cognoscitivas, inaugura, indispensablemente, otra praxis epistemológica, pedagógica y didáctica de esos profesionales, que los obliga a poner en tela de juicio aquello que fundamenta, su profesionalidad.

Parece ser claro lo que inaugura esa nueva praxis es entrar a pensar que una evaluación por competencias no puede seguir insistiendo en que los muchachos y muchachas repitan al pie de la letra las informaciones especializadas, de los contenidos curriculares; sino que a partir de ellas puedan interpretar, argumentar y proponer problemas, tanto de la vida cotidiana como de la especificidad del objeto de saber curricular en el interior del cual, y como pretexto, se propone la referida evaluación. En esto existe el acuerdo de que cualquier evaluación de este tipo debe suministrar a los evaluados los contextos teóricos y los datos que requieren para que desde sus interpretaciones idiosincráticas puedan resolver los interrogantes que se les plantean en dicho examen.

Se piensa que, en consecuencia, se hace indispensable reflexionar en torno al sentido que tiene una evaluación por competencia, dentro de lo dicho, en la medida en que tal evaluación dé al traste, por fin, con una praxis educativa en la que aquel que repite lo transmitido, es el que sabe y merece la mejor calificación. Por supuesto, es aquí en donde esta nueva praxis exige un cambio en las concepciones epistemológicas, pedagógicas y didácticas de profesores y profesoras, ya que el referente de la evaluación, deja de ser el texto aprobado por las respectivas comunidades de especialistas para desplazarse al 
examen crítico de si la interpretación, la argumentación y la propuesta de cada estudiante pueden ser admitidas según la logicidad histórica del saber objeto de estudio.

Dentro de esta reflexión, es en la que cabe las preguntas iniciales. Qué sentido tiene practicar una evaluación por competencias? ¿Desde qué modelo epistemológico, pedagógico y didáctico se formula y practica tal evaluación? ¿Qué resultados han de obtenerse con dicha evaluación? ¿Qué significan dichos resultados? ¿Qué hacer con ellos? ¿Cómo ellos se revierten en los términos de la fundamentación epistemológica, pedagógica y didáctica que direccionan un proyecto curricular centrado en la reconstrucción y construcción de competencias?

Se sostiene aquí que antes de cualquier ejercicio de cómo evaluar por competencias, es menester que cada institución educativa entre a decidir, de forma colectiva, cuáles son las competencias que han de direccionar su proyecto educativo institucional; decisión ésta en la cual cada uno de los colectivos de profesores, por áreas disciplinares, las formas cómo esas competencias se reflejan en cada uno de esas unidades didácticas. Se considera que, después de este ejercicio, que es fundamentalmente epistemológico, y no antes, es que hay que introducir a los estudiantes, para que, sin desconocerlos, se precisen las estrategias pedagógicas y didácticas que le posibiliten a ellos han de adentrarse en ese proceso de reconstrucción y construcción. Antes no.

Hay aquí una logicidad que afirma que si este proceso de reconstrucción y construcción no se ha dado comunitariamente, entonces resulta arbitrario pensar qué es lo que se va a evaluar, de qué manera se va a adelantar este proceso y cuáles son los fundamentos epistemológicos, pedagógicos y didácticos que sustentan esa evaluación. Dado que si esos pedagogos y didáctas en las disciplinas en que ejercen no explicitan en dichos sabores y en sus unidades didácticas, cómo se realiza en cada caso, las competencias, interpretar, argumentar y producir, entonces, no tienen bases para comprometerse en el problema de la reconstrucción y construcción de competencias.

\section{CONCLUSIONES}

Se ha tomado distancia en relación con las aproximaciones que reducen las competencias cognoscitivas a habilidades y destrezas. Se ha puntualizado, igualmente, en la distinción epistemológica, pedagógica y didáctica que hay que hacer entre objetivos y logros y entre estos y competencias, recalcándose que un proyecto curricular centrado en la reconstrucción y construcción de estas últimas obedece a otras intencionalidades educativas y formativas, las cuales reclaman su inscripción en otro concepto acerca de la evaluación de los aprendizajes.

En el intento de explicitación se ha propuesto que las competencias cognoscitivas se realizan en tres dimensiones: la cognoscitiva, la afectiva y la actuacional; a la vez que cada una de estas, para efectos pedagógicos y didácticos, se ha especificado en las siguientes subdimensiones: conceptual, metodológica, estética, actitudinal y axiológica. Se afirma que sólo después de que se ha llegado a la comprensión de cómo los contenidos curriculares se pueden exponer en esas dimensiones y subdimensienes, es factible entrar a discutir las estrategias pedagógicas y didácticas para adelantar una formación y educación consecuentes con tales presupuestos.

Se considera que son los profesores y profesoras como miembros de la comunidad de profesionales de la educación los que, en cada institución educativa, previa discusión 
sistemática y rigurosa, han de decidir cual es el trío de competencias que ha de direccionar sus actuaciones pedagógicas y didácticas. Para tal efecto ha de apoyarse en resultados de investigaciones en el campo, en publicaciones especializadas y adelantar intercambios con otros miembros de la comunidad que trabajan en dicho campo.

Son los profesores y profesoras, como comunidad de especialistas, los que han de discutir y tomar las decisiones.

\section{BIBLIOGRAFIA}

Ausubel, D.; Novak, J. D. y Hanesian H. 1983. Psicología educativa. Un punto de vista cognoscitivo. Segunda Edición. México, Trillas.

Chomsky, N. 1965. Aspects of theory of sintax. Cambridge, Mass: MIT Press.

Gallego Badillo, R. 1999. Competencias cognoscitivas. Un en foque epistemológico, pedagógico y didáctico. Santa Fe de Bogotá: Cooperativa Editorial Magisterio.

Gallego Badillo, R y Pérez Miranda, R. 1999. La construcción de competencias. Una intencionalidad curricular. Paradigma. Volumen XX, №. 1. 7-29.

1998. Aprendibilidad-enseñabilidad-educabilidad: una discusión. Revista Colombiana de Educación. Nos. 36-37,69-92.

Gallego Badillo, R., Pérez Miranda, R. y Torres de Gallego, L. N. 1997. El concepto de aprendizaje total. Una aproximación teórica. Estudios en pedagogía y didáctica. 2(1), 413.

Hayles, N. K. 1993. La evolución del caos.Barcelona:Gedisa.

Kuhn, T. S. 1972. La estructura de las revoluciones científicas. México: Fondo de Cultura Económica.

Piget, J. 1983. Teorías del lenguaje, Teorías del aprendizaje. El debate entre Jean Piaget y Noam Chomsky. Organizado y recopilado por Massimo Piattenelli-Palmarini. Barcelona: Editorial Crítica.

Prigogine, I. y Stengers, I. 1990. La nueva alianza. Metamorfosis de la ciencia. Madrid: Alianza.

Toulmin, S. 1972. La comprensión humana. Vol. 1. El uso colectivo y la evolución de los conceptos. Madrid: Alianza. 